\title{
Tagesklinische dialektisch-behaviorale Therapie: Eine Verlaufsuntersuchung nach Entlassung. Gibt es Prädiktoren für eine Verschlechterung?
}

\author{
Christoph Richter ${ }^{a, b}$ \\ ${ }^{a}$ Klinik für Psychiatrie, Psychotherapie und Psychosomatik, Vivantes Klinikum Kaulsdorf, Berlin, Deutschland; \\ ${ }^{b}$ Klinik für Psychiatrie und Psychotherapie, Charité Campus Mitte, Charité - Universitätsmedizin Berlin, Berlin, Deutschland
}

\section{Schlüsselwörter}

Dialektisch-behaviorale Therapie - Tagesklinik .

Borderline-Persönlichkeitsstörung · Follow-up · Prädiktoren .

Katamnese

\begin{abstract}
Zusammenfassung
Einleitung: Die dialektisch-behaviorale Therapie (DBT) für Patienten mit Borderline-Persönlichkeitsstörung (BPS) ist evidenzbasiert. Es gibt jedoch nur eine kleine Anzahl von Studien, die die Frage einer möglichen anhaltenden Wirkung von DBT untersuchen. Über Prädiktoren dieser Nachhaltigkeit ist wenig bekannt. Methode: Am Beispiel eines zertifizierten tagesklinischen Settings wird der posttherapeutische Verlauf untersucht. Mögliche Prädiktoren einer Verschlechterung nach Therapieende sollen erkannt werden. Hierzu wurden Patienten der DBT-Tagesklinik, deren Daten schon in eine prospektive, naturalistische, offene und nicht kontrollierte Vorstudie eingingen, erneut retrospektiv befragt. Somit konnten die Scores der Borderline-Symptomliste 95 (BSL-95) und des Beck-Depressions-Inventars (BDI) zu 3 verschiedenen Zeitpunkten (Z1 = Start, Z2 = Therapieende, Z3 = Katamnesezeitpunkt) verglichen werden. Der Fragebogen zu «Nebenwirkungen stationärer Psychotherapien" (NESTAP) lieferte weitere mögliche Prädiktoren. Ergebnisse: Der Katamnesezeitraum betrug 3-51 Monate. 82 Patienten wurden angeschrieben, es antworteten 41. Anhand des BDI und der BSL-95 (Differenz Z1 zu Z3, $p<0,05$ ) ist eine Symptombesserung in dieser Gruppe auch weit über das Therapieende feststellbar. In einem linearen Regressionsmodell mit einer Effektstärke von 1,04 (Cohen) zeigt eine Subgruppe (25\%), gekennzeichnet durch hohe Werte des Prädiktors "Dysphorie» im BSL-95 (Z2 zu Z1) eine geringe anhaltende Wirkung der DBT (Differenz der BSL-95- und BDI-Scores zu $Z 2$ und Z3). Schlussfolgerungen: Es konnten Hinweise für ein tagesklinisches DBT-Programm zur anhaltenden Symptombesserung bis 51 Monate nach Therapieende dargestellt werden. Für $50 \%$ der Patienten ist hierfür keine Aussage möglich. Auch gab es keine Kontrollgruppe. Die Veränderung von "Dysphorie» während der Therapiephase scheint diese therapeutische Wirkung über diesen Katamnesezeitraum zu beeinflussen. Weitere Studien sollten folgen, um die Daten zu prüfen oder andere Prädiktoren zu finden.
\end{abstract}

\section{Keywords}

Dialectical behavior therapy - Day clinic .

Borderline personality disorder - Follow-up - Predictor .

Sustainability

\section{Summary}

Follow-Up and Predictors of Deterioration in a Day Clinic

Dialectical-Behavior Therapy Setting: a Naturalistic Katamnestic Trial

Background: The dialectical-behavior therapy (DBT) is well evaluated for patients suffering from borderline personality disorder (BPS). Only a few studies exist that focus on a lasting effect of DBT in a katamnestic design. Very little is known about predictors of this sustainability. Method: First, a possible lasting effect of a certified 12-week day clinic setting is described. Second, possible predictors of deterioration will be identified. Patients who completely finished this day clinic DBT and whose data had already been used for a prospective, naturalistic, open and uncontrolled study were now retrospectively questioned. Thus, the scores of the Borderline Symptom List 95 (BSL-95) and the Beck's Depression Inventory (BDI) could be compared at 3 different time points ( $Z 1$ = start, $Z 2$ = end of therapy, $Z 3=$ catamnesis time). Other possible predictors were assessed by means of a questionnaire on the side-effects of inpatient psychotherapies (NESTAP). Results: The catamnestic period was 3-51 months. From 82 selected former patients, 6 men and 35 women responded. On the basis of the BDI and BSL-95, a lasting effect of DBT day clinic treatment can also be ascertained far beyond the end of therapy. A still existing improvement (BDI, BSL-95) at Z3 compared to Z1 is observed $(p<0.05)$. A subgroup $(25 \%)$, characterized by lower improvement of 'dysphoria' (subscale of BSL-95) over Z1 and Z2, shows a lower lasting effect based on differences in the BDI and BSL-95 data between Z2 and Z3 (Cohen's effect size = 1.04). Conclusions: This observational katamnestic design suggests a lasting effect of a day clinic DBT program for BPS for up to 51 months for respondents. However, no statement is possible regarding the non-responding patients (50\%). A control group would have shown an alternative or natural course. An improvement of 'dysphoria' during the therapy phase may influence the longer therapeutic sustainability. Further studies using a controlled design should follow in order to confirm or disprove these results or to find additional predictors of sustainability in DBT for BPS.

\section{KARGER}

() 2018 S. Karger AG, Basel
Dr. Christoph Richter, MBA Klinik für Psychiatrie, Psychotherapie und Psychosomatik Vivantes Klinikum Kaulsdorf

Myslowitzer Straße 45, 12621 Berlin, Deutschland christoph.richter@vivantes.de 


\section{Einleitung}

Die Borderline-Persönlichkeitsstörung (BPS) ist nicht die häufigste psychische Erkrankung. In Klinik und Praxis bestimmt sie aber oft den Alltag und erschwert erwünschte Therapieerfolge.

Die BPS ist vor allem durch eine Emotionsregulationsstörung charakterisiert. Das Leitsymptom sind Spannungszustände [Stiglmayr, 2003]. Diese Affekt- und Impulskontrollstörungen bedingen defizitäre Verhaltensweisen, die, wie im Diagnostic and Statistical Manual of Mental Disorders (DSM)-5 (ebenfalls im früheren DSM-IV) definiert, zu einem tiefgreifenden Muster von Instabilität $\mathrm{zu}$ Beginn des frühen Erwachsenenalters führen, und es müssen mindestens 5 von 9 Kriterien vorliegen.

In 2 repräsentativen epidemiologischen Befragungen wird eine eher konservative Prävalenz von $0,7 \%$ in der Bevölkerung beschrieben [Coid et al., 2006; Torgersen et al., 2000]. Das lässt in Deutschland mindestens 570000 betroffene Menschen annehmen. Zusätzlich hat die BPS eine hohe Komorbidität anderer psychischer Erkrankungen (Depressionen, Suchterkrankungen usw.) [Trull et al., 2010; Zanarini et al., 1998]. Vier Milliarden Euro primärer Gesamtkosten, dies entspricht etwa $20 \%$ der für alle psychischen Störungen verwandten finanziellen Mittel in Deutschland, werden der BPS zugerechnet [Bohus und Kröger, 2011].

Trotz therapeutischer Erfolge mit Verbesserungen für die Lebensqualität gelingt es der Hälfte der Menschen mit BPS nicht, ein Beschäftigungsverhältnis zu erreichen, viele werden auch frühzeitig berentet [McMain et al., 2012]. Die Frage, inwieweit gegebenenfalls für eine Untergruppe der BPS eine Heilung gelingen kann, bleibt wenig fundiert, da der Beobachtungszeitraum noch zu gering ist, um Verbesserungen in den «key life areas» nachweisen zu können [Stone, 2016].

Spezifische medikamentöse Therapieansätze gibt es derzeit nicht, vielfältige Substanzklassen finden außerhalb der Zulassung symptombezogen und oft in Polypharmazie aufgrund hoher Akuität Anwendung [Lieb et al., 2010; Sansone und Wiederman, 2009].

Als eine Psychotherapieform zur Behandlung der BPS ist die dialektisch-behaviorale Therapie (DBT) nach M. Linehan am besten evidenzbasiert und durch vielfältige kontrollierte Studien belegt [Bohus and Kröger, 2011; Cristea et al., 2017; Pasieczny und Connor, 2011]. Im (teil)stationären Sektor hat sich historisch in Deutschland die DBT zur am weitest verbreiteten störungsspezifischen Methode der BPS entwickelt. Für diese Therapieform sind auch Effektivität und Effizienz evidenzbasiert am sichersten nachgewiesen [Stoffers et al., 2012].

Tageskliniken spielen bisher nur eine geringe Rolle bei der Behandlung der BPS [Kallert et al., 2003], obwohl diese als Baustein zwischen ambulanter und stationärer Therapie alltagsnäher und mit weniger Regression und Hospitalismus behaftet sind sowie seltener ein Herausfallen aus sozialen Netzen zur Folge haben [Eikelmann, 2010].

Bisherige Studien zur Wirksamkeit der DBT zeigen klassischerweise nachhaltige Effekte hinsichtlich der Abnahme von Suizidalität, Selbstverletzung und Klinikeinweisung [Kienast et al., 2014; Stiglmayr et al., 2014a] sowie Kosteneffizienz [Kliem et al., 2010;
Priebe et al., 2012; van den Bosch et al., 2014]. Kleindienst et al. [2008] stellten in einer kontrollierten Studie (19 Patientinnen der Wartegruppe vs. 31 Patientinnen einer 4-monatigen stationären DBT) dar, dass DBT signifikant die Borderline-typische Symptomatik verbesserte, während in der Wartegruppe keine Veränderung gemessen werden konnte. So konnten sich $62 \%$ der DBTGruppe, aber nur 31\% der Wartegruppe vom selbstverletzenden Verhalten abhalten.

In einer katamnestischen Nachbefragung 6 Monate nach einem 12-wöchigen stationären DBT-Programm für Patienten mit einer BPS konnte eine nachhaltige Effektivität anhand einer hochsignifikanten Reduktion um 10 Punkte auf der Beck-DepressionsInventar(BDI)-Skala festgestellt werden. Nur 1 von 21 Patienten zeigte eine signifikante Verschlechterung des durchschnittlichen Belastungsindexes anhand der Symptom-Checkliste (SCL)-90-R über diesen Zeitraum. Patienten, die mithilfe auch weiter ambulant durchgeführter Skills ihr Emotionsmanagement trainierten, profitierten weiterhin vom positive Effekt dieser DBT [Dams et al., 2007].

Untersuchungen zur langfristigen Recovery oder Remission der Symptomatik von BPS liegen kaum vor. Eine Fallserie [Stone, 2017] konnte zeigen, dass von 40 Patientinnen mit diesem beschriebenen Störungsbild zwischen 25 und 50 Jahre nach Diagnostik und nicht näher beschriebener Psychotherapie etwa zwei Drittel ein Level klinischer Remission bzw. Recovery erreichten. Alvarez-Tomás et al. [2017] beschrieben in einer 10-jährigen naturalistischen, prospektiven Studie an 64 Patienten (56 Frauen) mit BPS, die vor 10 Jahren ambulante DBT erhielten, dass etwa die Hälfte nicht mehr die Mindestanzahl von 5 DSM-Kriterien zeigte und die Hälfte nicht stationär-psychiatrisch behandelt werden musste, jedoch $75 \%$ weiterhin Psychotherapie erhielten und 77,5\% ambulant psychiatrisch betreut waren. Die Notwendigkeit sozialer Assistenz stieg in diesem Zeitraum von etwa $26 \%$ auf zuletzt $45 \%$ wiederum an.

Gunderson et al. [2011] zeigten bei 175 BPS-Patienten über einen 10-Jahres-Zeitraum in einem multizentrischen, naturalistischen, longitudinalen Studiendesign mit wiederholten Messzeitpunkten, dass $85 \%$ der BPS-Patienten remittierten, jedoch weiterhin schwere und persistierende soziale Funktionseinbußen aufwiesen.

Nur wenige Studien untersuchen eine anhaltende Wirkung von DBT z.B. über mehr als 6 oder gar 12 Monate [Kleindienst et al., 2008; Kröger et al., 2014; Linehan et al., 2015; Rüsch et al., 2008; Stiglmayr et al., 2014b; Swales et al., 2012].

So finden sich eher Untersuchungen zum Drop-out-Risiko als zu Prädiktoren einer anhaltenden Wirkung. Kröger et al. [2014] zeigten in einer Nachuntersuchung von 541 Patienten mit BPS in einer 3-monatigen stationären DBT, dass $14 \%$ der Patienten die Therapie vorfristig beendeten. Gründe waren Motivationseinbruch und geringe Toleranz gegenüber emotionalem Disstress. Außerdem waren diese Abbrüche mit Anorexia nervosa, Suizidversuchen und Alkoholkonsum assoziiert. Rüsch et al. [2008] beschrieben in einem nicht kontrollierten Design stationärer DBT für 60 Frauen, dass Vermeidung und Angst zu Beginn der Therapie Prädiktoren eines frühzeitigen Abbruchs darstellen. Insgesamt konnten Kröger 
et al. zeigen [2006, 2014], dass mit Drop-out-Raten in einer stationären DBT von bis zu 32\% zu rechnen ist. Mögliche Prädiktoren wurden nicht beschrieben. Erhöhte Abbruchraten bis zu 51\% in einer ambulanten DBT (ohne Kontrollgruppe) zeigten sich in einer Kohorte von 56 BPS-Patienten bei einem höheren Basislevel von Disstress sowie Nicht-Akzeptanz der Emotionalität [Landes et al., 2016].

Als Prädiktoren für geringe Ansprechbarkeit gelten komorbide Essstörungen, posttraumatische Belastungsstörungen (PTBS) und Suchterkrankungen [Kienast et al., 2014]. Aber auch in einer randomisierten kontrollierten Studie reduzierten komorbide Dissoziationen während der DBT bei BPS und gleichzeitig vorliegender PTBS deren Therapieerfolg [Kleindienst et al., 2016]. Bedics et al. [2015] konnten an 101 Frauen mit einer BPS über eine 1-JahresSpanne zeigen, dass die Stärke der therapeutischen Allianz die Suizidalität beeinflusst und damit auch den Therapieerfolg. Diese Ergebnisse konnten auch Steuwe et al. [2017] replizieren.

Links et al. [2013] untersuchten das Suizidrisiko von 108 Teilnehmern eines 1-jährigen DBT-Programms. Risikofaktoren erneuter Suizidversuche in einem 2-Jahres-Zeitraum waren sexuelle Kindheitstraumata und die Anzahl an Krankenhausaufenthalten sowie die Anzahl an Suizidversuchen zu Beginn der Therapie. In einer Gruppe ambulanter BPS-Patienten $(n=447)$ eines KurzzeitDBT-Programms mit Wiederholung nach 1 Jahr wird eine geringe Schulausbildung als Prädiktor für vorzeitige Abbrüche beschrieben [Perroud et al., 2010]. Auch in einer 15-monatigen Katamneseuntersuchung von Kröger et al. [2010] an 24 Frauen mit BPS bei allerdings komorbider Anorexia bzw. Bulimia nervosa beschränkten sich die Autoren auf den Nachweis ausgewählter anhaltender Effekte, jedoch werden keine Prädiktoren hierfür beschrieben.

Hinsichtlich der Frage, welche Prädiktoren anhaltende Effekte von DBT nun bestimmen könnten, beschreiben Perroud et al. [2010] in einer Nachuntersuchung im Zuge einer DBT-Wiederholung nach 1 Jahr bei 447 ambulanten Patienten, dass hohe schizoide bzw. niedrige narzisstische Merkmale, aber auch Frühberentung die DBT-Wirkdauer nach 1 Jahr reduzieren. In einer anderen Untersuchung eines nur 5-tägigen DBT-Programms wurden 47 Teilnehmerinnen nach 3 Monaten erneut in einem unkontrollierten naturalistischen Studiendesign befragt. Prädiktoren einer geringeren anhaltenden Effektivität waren hierbei Identitätsstörungen und die Angst vor dem Verlassenenwerden [Yen et al., 2009].

Insgesamt ist die Studienlage möglicher Prädiktoren hinsichtlich der anhaltenden Wirkung der DBT bzw. Risikofaktoren einer Verschlechterung nach Therapie noch wenig belastbar, zumeist fokussieren diese auf das Abbruchrisiko und soziodemografische oder psychische Merkmale zur Vorhersage für ein unmittelbares Therapieergebnis zum Therapieende [Kröger et al., 2013].

Dies war Anlass, Patienten einer naturalistischen Vorstudie katamnestisch erneut zu befragen. In der Vorstudie konnte zuvor für diese Gruppe eine Symptomverbesserung in einem 12-wöchigen tagesklinischen DBT-Setting nachgewiesen werden. Eingeschlossen waren hierbei 31 Fälle. Eine Verbesserung im BDI-Score um $50,8 \%(\mathrm{p}<0,001)$, in der SCL-90 um 42,9\% ( $<<0,01)$ und in der Borderline-Symptomliste 95 (BSL-95) um 48,4\% ( $<<0,001$ ) bei einer Power von 0,99 wurden gezeigt. Es fand sich kein Zusammenhang zwischen Medikationsänderung und Symptomreduzierung [Richter et al., 2014].

\section{Methode}

\section{DBT in der Tagesklinik}

In der dargestellten Untersuchung soll der Symptomverlauf nach einer 12-wöchigen DBT einer tagesklinischen Patientenpopulation geprüft werden. Die hier beispielhaft verwandte Tagesklinik ist nach den Richtlinien des Dachverbands Dialektisch Behaviorale Therapie e.V. zertifiziert. Sie ist im Landeskrankenhausplan von Berlin aufgenommen, sodass nach $₫ 108$ des Fünften Buches Sozialgesetzbuch (SGB V) die gesetzlichen Krankenkassen die Behandlungskosten übernehmen. Eine tagesklinische Behandlung steht zwischen einer ambulanten und stationären Therapie. Die ambulante Behandlung hat Vorrang vor der stationären Behandlung, wenn das Behandlungsziel zweckmäßig und ohne Nachteil für die Patientin oder den Patienten mit den Mitteln der ambulanten Versorgung erreicht werden kann ( $\$ 39$ Absatz 1 Satz 2 SGB V). Die Aufnahme der Patienten in die hier untersuchte Tagesklinik erfolgte nach ärztlicher Indikationsprüfung mit ambulanter fachärztlicher Einweisung und regelhafter Nachprüfung durch den Medizinischen Dienst der Krankenkassen. Im Vorfeld waren die Mittel der ambulanten Versorgung für diese Patientenpopulation nicht mehr als ausreichend betrachtet worden. So wünschten Einweiser wie auch die Patienten eine intensive und spezifische Therapie, entweder weil ambulante Psychotherapeuten die Patienten als zu akut beschrieben oder die ambulante Psychotherapie in der Intensivität als zu gering oder unspezifisch erachtet wurde oder wiederum weil Patienten aus verschiedensten Gründen keinen Therapieplatz erhielten und so die Möglichkeit nutzten, innerhalb einer Wartezeit zwischen etwa 6 und 12 Wochen in diese Tagesklinik aufgenommen zu werden. Gleichzeitig war das Interesse groß, weniger aus den sozialen Bezügen zu fallen und auch zuhause zu wohnen, was bei einer vollstationären Therapieoption nicht möglich gewesen wäre. Teilweise lagen Empfehlungen von Akutkliniken vor, Direktverlegungen erfolgten jedoch nicht.

In der Tagesklinik wurden folgende Therapiekomponenten angeboten: DBT-Gruppen (Skillstraining, 2 h pro Woche: Stresstoleranz, Emotionsregulation, soziale Kompetenz usw.), Einzeltherapie (1 h/Woche), Co-TherapeutenEinzel (praktische Umsetzung von Skills/Inhalten, 1 h/Woche), Psychoedukation (Basisgruppe 1,5 h/Woche), Bezugsgruppe (Peer Group, etwa 3 h/Woche), Achtsamkeitsgruppe (1 h/Woche), Körpertherapie (Gruppe und/oder individuell, 1-2 h/Woche), Teambesprechung/Supervision für Therapeuten (2 h/Woche) sowie die 2-wöchentliche Oberarztvisite. Die DBT-Gruppen (Skillstraining), Einzeltherapie und Teambesprechungen/Supervision erfolgten nach dem DBT-Manual von Linehan. Acht h Teamschulung pro Jahr werden durch einen anerkannten externen DBT-Supervisor erbracht. Zusätzlich findet vor allem über 4-5 h/Woche interaktionelle Ergotherapie als Kochgruppe statt. Außerdem sind etwa $2 \mathrm{~h}$ für die Vor- und Nachbearbeitung der Therapie (Hausaufgaben, eigenständiges Skillstraining) für die Patienten reserviert. Somit stehen dem $\mathrm{Pa}$ tienten direkt mindestens $15 \mathrm{~h}$ Therapie über 12 Wochen (insgesamt also etwa 180 Therapiestunden zur Verfügung). Dies sind ungefähr $100 \mathrm{~h}$ mehr als in einem ambulanten Setting in Deutschland zumeist möglich ist.

Bei einem für die Zertifizierung nachzuweisenden Personalschlüssel von mindestens $80 \%$ nach der Verordnung über Maßstäbe und Grundsätze für den Personalbedarf in der stationären Psychiatrie stehen 2 ausgebildete DBT-Therapeuten (1 Facharzt für Psychiatrie und Psychotherapie und 1 approbierte Diplom-Psychologin), 1 psychologische DBT-Therapeutin in Ausbildung und mindestens 2 DBT-Pflegekräfte/Fachtherapeuten zur Verfügung. Insgesamt wird so Adhärenz gegenüber den klassischen DBT-Techniken gewährleistet [Lynch et al., 2007]. Ein Drittel der 20 Behandlungsplätze wird für die Therapie von BPSPatienten reserviert. Eine getrennte etwa 2-wöchige Diagnostikphase im Vorfeld wird unabhängig von der 12-wöchigen Therapiedauer vorgehalten. Die übrigen Krankheitsbilder stellen affektive und Angsterkrankungen, die ein eigenständiges, von der zertifizierten DBT unterschiedenes Therapieprogramm erhalten. Von Montag bis Freitag (8:30-16:00 Uhr) wird das DBT-Programm in dieser 
Tagesklinik durchgeführt. Am Wochenende ist die Tagesklinik geschlossen, eine Notversorgung erfolgt über die Klinik [Richter et al., 2014]. Im Gegensatz dazu erfolgt eine ambulante Psychotherapie üblicherweise nur je 1-2 h pro Woche. Eine stationäre (Psycho-)Therapie bietet ein Angebot in einem therapeutischen Milieu über $24 \mathrm{~h}$ an 7 Tagen die Woche an. Diese Tagesklinik mit DBT-Modul bietet hiermit also die wesentlichen Elemente eines therapeutischen Milieus an: Diagnosebestätigung, intensive Therapie, einen therapeutischen Schutzraum, die Möglichkeit der Tagesstrukturierung, ein Übungsfeld, kontinuierliche Motivationsarbeit, Modellernen vom Therapeuten und von Mitpatienten sowie funktionalere soziale Kontaktmöglichkeiten [Linden, 2014].

\section{Design der Studie}

Daten einer prospektiven, naturalistischen, offenen und nicht kontrollierten Vorstudie [Richter et al., 2014] konnten als Basis verwendet werden. In dieser Vorstudie füllten die Patienten zum Therapiestart in der 1. Woche (Zeitpunkt 1) und kurz vor Therapieendende in der 11. Woche (Zeitpunkt 2) das BDI [Hautzinger et al., 1995] und die BSL-95 [Zentralinstitut für Seelische Gesundheit (ZfS), 2014] aus, die auch für die Nachuntersuchung Verwendung fanden. Aus dieser Vorstudie waren der Schulabschluss und die Mindestanzahl an Suizidversuchen vor dem Zeitpunkt 1 bekannt. Fragebögen plus strukturierte Interviews (DSM-IV) wurden jeweils von intensiv supervidierten Psychotherapeutinnen in Ausbildung oder von den approbierten DBT-Therapeuten der Tagesklinik durchgeführt.

Hierbei wurden zuvor alle Patienten ausgeschlossen, die nicht mindestens 5 Kriterien der BPS nach dem DSM-IV erfüllten (zum damaligen Zeitpunkt lag das DSM-5 noch nicht vor) und vorfristig das 12-wöchige tagesklinische DBTTherapieprogramm beendeten. Weiterhin mussten die Voraussetzungen für eine tagesklinische Behandlung vorhanden sein. Die BPS stellte die Hauptdiagnose, andere mögliche Nebendiagnosen durften nicht akut stationär behandlungsbedürftig sein oder im Vordergrund stehen.

Die vorliegende Folgeuntersuchung ist somit eine naturalistische, nicht kontrollierte retrospektive Datenerhebung zur Qualitätssicherung.

Die Nachbefragung erfolgte freiwillig im Rahmen der gesetzlich festgeschriebenen Qualitätssicherung und -weiterentwicklung von Krankenhäusern in Deutschland ( $\$ 135 \mathrm{a}$ Abs. 1 SGB V). Somit war die Einholung eines Ethikvotums in diesem besonderen Fall nicht notwendig. Alle angeschriebenen und rückantwortenden Patienten willigten einem «informed consent» ein. Die Patienten erhielten hierzu im informativen Anschreiben eine schriftliche Aufklärung zum Befragungszweck, zur Pseudonymisierung und zur Sicherheit der Datenverarbeitung. Das unbedingte Freiwilligkeitsprinzip wurde strikt beachtet und der Datenschutzbeauftragte des Krankenhauskonzerns Vivantes intensiv mit einbezogen. Es wurde darauf hingewiesen, dass es sich nicht um eine Fortsetzung der Behandlung handele. Sämtliche Tests stellen Routinemessinstrumente zur Diagnostik und Erfolgskontrolle dar und wurden während der Therapiephase mit den Patienten besprochen. Den Patienten wurde versichert, dass ein Nachteil bei Nichtbeteiligung dieser katamnestischen Befragung nicht zu befürchten ist. Auch wurden die Kriterien der Helsinki-Deklaration von 1975 beachtet, soweit diese relevant waren. Die Datensätze wurden pseudonymisiert, ein individueller Bezug ist somit nicht mehr erkennbar. Die Tagesklinik setzt damit eine wissenschaftliche Überprüfung ihres Behandlungskonzeptes und der Behandlungsergebnisse um.

Im Rahmen der vorliegenden Nachuntersuchung wurden die Anzahl von Suizidversuchen im Zeitraum 2 und 3 und ausgewählte Parameter vom NESTAP (Fragebogen: «Nebenwirkungen stationärer Psychotherapien») erhoben [Brakemeier et al., 2012]. Der Fragebogen NESTAP erfasst retrospektiv mögliche Einflussfaktoren auf subjektiv negativ erlebte Wirkungen der Therapie. Angeschrieben wurden nur die Patienten, die obige Einschlusskriterien erfüllten.

Für den Katamnesezeitraum konnten nur Angaben der Patienten zur Art einer möglichen Anschlussbehandlung erfasst werden, so erfolgten auch kein Zugriff auf Krankenkassendaten, keine Nachexploration und kein Kontakt mit ärztlichen oder psychotherapeutischen Behandlern.

Die Auswertung am Ende der Rekrutierungsphase erfolgte pseudonymisiert. Die Daten wurden im Sinne einer As-Treated-Analyse ausgewertet. Die Datenqualität war zumeist sehr hoch, nur in sehr wenigen Fällen fehlten Testungen zu einem bestimmten Zeitpunkt. Dies erklärt die unterschiedliche Stichprobenan- zahl. Zur Prüfung auf Normalverteilung und Varianzhomogenität der Daten wurden der Kolmogorow-Smirnow- und der Levene-Test eingesetzt. Weiterhin fanden der gepaarte T-Test für abhängige Stichproben als Mittelwertsvergleich und die Darstellung von Häufigkeiten mit prozentualer Beschreibung im Rahmen der deskriptiven Statistik Anwendung.

Zur Prüfung eines Zusammenhangs zwischen möglichen Prädiktoren (Geschlecht, Alter, Zeitdauer zwischen Therapieende und Katameneszeitpunkt, Anzahl der DSM-IV-Kriterien, absolvierte Psychotherapie oder ärztliche Kontakte nach Ende der Therapiephase) und Skalenveränderungen (BDI, BSL-95 bzw. deren Subskalen wie z.B. Autoagressivität, soziale Isolation, Feindseligkeit und andere als abhängige Variablen) wurde für die Nominalskalen Geschlecht und anschließende Psychotherapie eine einfache Varianzanalyse mit Messwiederholungen durchgeführt. Für die metrisch abhängigen Variablen konnte die lineare bzw. in einem weiteren Schritt die multiple Regression genutzt werden. Zuvor fand die Prüfung ihrer Voraussetzungen statt (Linearität des Zusammenhangs und der Koeffizienten, bedingter Erwartungswert, Homoskedastizität, Unabhängigkeit und Normalverteilung des Fehlerwertes). Das Statistikprogramm SPSS 25 wurde genutzt. Die Modellgüte bzw. Effektstärke konnten ermittelt werden.

Hauptpostulat ist, dass eine teilstationäre DBT-Effektivität auch nachtherapeutisch erhalten bleibt. Zusätzlich sollen mögliche Prädiktoren (siehe oben) für abhängige Variablen wie BDI- und BSL-95-Scores geprüft werden.

\section{Ergebnisse}

\section{Normalverteilung und Soziodemografie}

Von allen bisher behandelten, insgesamt 98 ehemaligen Patienten der Vorstudien (11 Männer, 87 Frauen) des zertifizierten DBTProgramms - davon brachen 16 Patienten die Therapie vor dem geplanten Therapieabschluss ab (zumeist ohne Angabe von Gründen, 2-mal wegen somatischer Komorbidität, 3-mal wegen einer Verlegung auf Station aufgrund eines schweren Suizidversuchs) wurden die verbliebenen 82 Patienten angeschrieben. Es antworteten 35 Frauen (85\%) und 6 Männer. Damit betrug die Rückantwortquote genau $50 \%$.

Der Katamnesezeitraum wird vom Therapieende bis zum Zeitpunkt der Befragung definiert, die zwischen Juni 2014 und Ende März 2015 erfolgte (1-malig wurde erinnert, wenn initial keine Rückantwort erfolgte). Der Katmnesezeitraum beträgt 3-51 Monate nach Entlassung (Mittelwert $=22,6$ Monate, Standardabweichung = 14,5 Monate). Etwa ein Drittel der Patienten verteilen sich auf die ersten 10 Monate des Katamnesezeitraumes, ein weiteres Drittel zwischen 11 bis 30 Monaten, das letzte Drittel auf den Zeitraum danach.

Hinsichtlich des Schulabschlusses charakterisiert sich die Untersuchungsgruppe wie folgt: Ohne Abschluss waren 2 (4,9\%), mit (erweitertem) Hauptschulabschluss 4 (9,8\%), mit Realschulabschluss 17 (41,5\%) und mit Abitur 13 (31,7\%) der Patienten; ohne Angabe blieben 5 (12,2\%). Weitere Anamnesedaten und Parameter, die möglicherweise Einfluss auf die Nachhaltigkeit haben könnten, sind in den Tabellen 1-3 abgebildet.

Der Kolmogorow-Smirnow-Anpassungstest wies für die Analyse der Testungen BDI und BSL-95 eine asymptomatische Signifikanz zwischen 0,167 und 0,200, also eine Normalverteilung der einzelnen Gruppenwerte nach. Unter dieser Voraussetzung konnten wir mithilfe der deskriptiven Statistik die Häufigkeiten ermitteln und Mittelwertanalysen durchführen. 
Tab. 1. Basisdaten

\begin{tabular}{lcrrrr}
\hline & N & M & SD & Minimum & Maximum \\
\hline Alter bei Therapiestart & 41 & 32,9 & 8,5 & 19 & 51 \\
Therapiezeitraum, Tage & 41 & 83,2 & 18,7 & 21 & 128 \\
Anzahl erfüllter DSM-IV-Kriterien & 41 & 6,3 & 1,1 & 5 & 9 \\
Mindestanzahl der Suizidversuche & 34 & 1,7 & 3,5 & 0 & 20 \\
\hline
\end{tabular}

$\mathrm{M}=$ Mittelwert; $\mathrm{SD}=$ Standardabweichung; $\mathrm{DSM}=$ Diagnostic and Statistical Manual of Mental Disorders.

Tab. 2. Art der Weiterbehandlung

\begin{tabular}{llrr}
\hline Art der Weiterbehandlung & & Häufigkeit & Prozent \\
\hline Ambulante Psychotherapie & ja & 28 & 68,3 \\
& nein & 11 & 26,8 \\
& ohne Angaben & 2 & 4,9 \\
Art der Psychotherapie & DBT & 11 & 26,8 \\
& VT & 12 & 29,3 \\
& andere Art der Psychotherapie & 1 & 2,4 \\
& nicht näher bekannt & 2 & 4,9 \\
& mehrere Antworten & 2 & 4,9 \\
& ohne Angaben & 13 & 31,7 \\
Ärztliche Anbindung & Hausarzt & 31 & 75,6 \\
& Psychiater & 7 & 17,1 \\
& unbekannt & 1 & 2,4 \\
Kontakt zu (teil)stationären & ohne Angaben & 2 & 4,9 \\
Einrichtungen & Rettungsstelle & 1 & 2,4 \\
& vollstationär Psychiatrie & 4 & 9,8 \\
& Krisendienst & 3 & 7,3 \\
& andere Tagesklinik & 3 & 7,3 \\
& mehrere Antworten & 6 & 14,6 \\
& ohne Antworten & 24 & 58,6 \\
\hline
\end{tabular}

DBT = Dialektisch-behaviorale Therapie; VT = Verhaltenstherapie

Tab. 3. Vergleich der Zeitpunkte 1,2 und 3

\begin{tabular}{|c|c|c|c|c|c|c|c|c|c|c|}
\hline & & \multirow[t]{3}{*}{ Einzelmittelwert $^{\mathrm{a}}$} & \multicolumn{5}{|c|}{ Paarige Differenzen } & \multirow[t]{3}{*}{$\mathrm{T}(\mathrm{M})$} & \multirow[t]{3}{*}{$\mathrm{df}(\mathrm{SD})$} & \multirow{3}{*}{$\begin{array}{l}\sigma(2 \text {-seitig }) \\
\text { Standardfehler } \\
\text { von M }\end{array}$} \\
\hline & & & \multirow[t]{2}{*}{ M } & \multirow[t]{2}{*}{ SD } & \multirow{2}{*}{$\begin{array}{l}\text { Standard- } \\
\text { fehler von M }\end{array}$} & \multicolumn{2}{|c|}{ 95\%-KI der Differenz } & & & \\
\hline & & & & & & Unterer & Oberer & & & \\
\hline Paar 1 & $\begin{array}{l}\text { BDI Z2 - } \\
\text { BDI Z3 }\end{array}$ & $22,6224,21$ & $-1,588$ & 10,425 & 1,788 & $-5,226$ & 2,049 & $-0,888$ & 33 & 0,381 \\
\hline Paar 2 & $\begin{array}{l}\text { BDI Z1 - } \\
\text { BDI Z3 }\end{array}$ & $32,0824,39$ & 7,694 & 13,94 & 2,323 & 2,978 & 12,411 & 3,312 & 35 & 0,002 \\
\hline Paar 3 & $\begin{array}{l}\text { BSL Z2 - } \\
\text { BSL Z3 }\end{array}$ & $\begin{array}{l}125,75(19 \%) \\
153,66(29 \%)\end{array}$ & $-27,906$ & 79,449 & 14,045 & $-56,551$ & 0,738 & $-1,987$ & 31 & 0,056 \\
\hline Paar 4 & $\begin{array}{l}\text { BSL Z1 - } \\
\text { BSL Z3 }\end{array}$ & $\begin{array}{l}190,3(47 \%) \\
157,03(31 \%)\end{array}$ & 33,267 & 82,18 & 15,004 & 2,58 & 63,953 & 2,217 & 29 & 0,035 \\
\hline
\end{tabular}

${ }^{a}$ In Klammern Angabe Prozentwert gleich oder höher zur Normierungsstichprobe.

$\mathrm{M}$ = Mittelwert; $\mathrm{SD}$ = Standardabweichung; KI = Konfidenzintervall; BDI = Beck-Depressions-Inventar; BSL = Borderline-Symptomliste 95; Z = Zeitpunkt.

Nachtherapeutische Symptomatik und Follow-up

Es zeigte sich, dass es zu einer leichten Verschlechterung des BDI-Scores zum Zeitpunkt 3 gegenüber dem Zeitpunkt 2 kam, jedoch ist hierfür keine Signifikanz auf dem 5\%-Niveau darstellbar. Eine Verbesserung der subjektiven depressiven Symptomatik (BDI) und der Borderline-typischen Symptomatik (BSL-95) zum Zeitpunkt 3 gegenüber dem Zeitpunkt 1 ist jedoch signifikant
(Tab. 3). Damit wird eine nachhaltige Symptomverbesserung innerhalb dieser untersuchten und zur Auswertung gekommenen Studienpopulation (ohne Vergleichsgruppe) beschrieben. Somit können die Ergebnisse der Gruppe um Kleindienst et al. [2008] untermauert werden, die nach 3-monatiger stationärer DBT deren nachhaltige Wirkung über 21 Monate in einem naturalistischen Follow-up-Design zeigten. 
Tab. 4. Einfluss der «Dysphorie»-Verbesserung auf die Differenz BDI Z2 minus BDI Z3, ANOVA

Tab. 5. Einfluss der «Dysphorie»-Verbesserung auf die Differenz BDI Z2 minus BDI Z3, Koeffizient

Tab. 6. Einfluss der «Dysphorie»-Verbesserung auf die Differenz BSL-95 Z2 minus BSL95 Z3, ANOVA

\begin{tabular}{llrrr}
\hline Modell & Quadratsumme & df & Mittel der Quadrate & F \\
\hline Regression & 25103,202 & 1 & 25103,202 & $\sigma, 876$ \\
Nichtstandardisierte Residuen & 66086,738 & 26 & 2541,798 & \\
Gesamt & 91189,940 & 27 & & \\
\hline
\end{tabular}

BDI = Beck-Depressions-Inventar; Z = Zeitpunkt; ANOVA = Varianzanalyse (analysis of variance).

\begin{tabular}{llllr}
\hline Modell & Regressionskoeffizient B & Standardfehler & $\beta$ & $\mathrm{T}$ \\
\hline (Konstante) & 9,336 & 13,751 & 0,679 & 0,503 \\
Differenz Dysphorie Z1 - Z2 & $-3,745$ & 1,192 & $-0,525$ & 3,143 \\
\hline
\end{tabular}

BDI = Beck-Depressions-Inventar; $\mathrm{Z}=$ Zeitpunkt.

\begin{tabular}{lcccc}
\hline Modell & Quadratsumme & df & Mittel der Quadrate & F \\
\hline Regression & 42403,608 & 1 & 42403,608 & 8,998 \\
Nichtstandardisierte Residuen & 117812,910 & 25 & 4712,516 & 0,006 \\
Gesamt & 160216,519 & 26 & & \\
\hline
\end{tabular}

BSL-95 = Borderline-Symptomliste 95; Z = Zeitpunkt; ANOVA = Varianzanalyse (analysis of variance).

Tab. 7. Einfluss der «Dysphorie»-Verbesserung auf die Differenz BSL-95 Z2 minus BSL$95 \mathrm{Z3}$, Koeffizient

\begin{tabular}{llcrr}
\hline Modell & Regressionskoeffizient B & Standardfehler & $\beta$ & $\mathrm{T}$ \\
\hline (Konstante) & 22,211 & 19,203 & 1,157 & 0,258 \\
Differenz Dysphorie Z1 - Z2 & $-4,907$ & 1,636 & $-0,514$ & 3,000 \\
\hline
\end{tabular}

BSL-95 = Borderline-Symptomliste 95; Z = Zeitpunkt.

\section{Prädiktoren}

Um mögliche Einflussgrößen für diese Veränderung herauszufinden, sollten Varianzen und gegebenenfalls ein Vorhersagemodell gefunden werden. Nach Feststellung der Varianzhomogenität sind die Varianzen mithilfe der Varianzanalyse (ANOVA) im BSL95 und BDI jeweils zum Zeitpunkt 3 nicht durch das «Geschlecht» erklärbar. Auch Parameter vom NESTAP, ob und welche Art einer Psychotherapie im Anschluss aufgenommen wurde oder ob ärztliche Kontakte zwischen den Zeitpunkten 2 und 3 bestanden, konnten die Varianzen in den BSL-95- und BDI-Scores (Zeitpunkt 3) nicht beschreiben.

Folgende weitere unabhängige Variablen bzw. mögliche Prädiktoren für die Nachhaltigkeit wurden zusätzlich untersucht: Dauer zwischen dem Ende der Therapie und dem Katamnesezeitpunkt, Anzahl der DSM-IV-Kriterien zu Beginn der Therapie und Subskalen des BSL-95 zum Therapiestart im Sinne besonderer Ausprägungen der BPS. Hinsichtlich der anhaltenden Wirkung wurde die Differenz der BSL-95-Werte bzw. des BDI-Scores zwischen Zeitpunkt 2 und 3 gemessen. Eine lineare und eine multiple Regressionsanalyse wurden durchgeführt.

Einzig die 2 folgenden Modelle der linearen Regressionsanalyse erbrachten signifikante Ergebnisse. Zum einen hat die Verbesserung von Dysphorie (Subskala des BSL-95) während der Therapiephase einen Einfluss auf die anhaltende Wirkung im Sinne einer geringeren subjektiven Depressivität (Differenz des BDI-Scores zum Zeitpunkt 2 und Zeitpunkt 3), F(1,26) = 9,876; $\mathrm{p}=0,004$; $\mathrm{n}=$ 28. Steigt also die Verbesserung der Dysphorie um 1 Einheit, erhöht sich die anhaltende Wirkung bezüglich der Depressivität (BDI) um 3,7 Einheiten zum Katamnesezeitpunkt auch nach mehreren Monaten. Nur 25\% der Streuung wird durch «Dysphorie» in diesem Modell erklärt, was nach Cohen jedoch einem starken Effekt von 1,04 entspricht (siehe Tab. 4 und Tab. 5).

Ähnlich zeigt sich das Modell der linearen Regression hinsichtlich der Verbesserung der Dysphorie in der Therapiephase in Bezug auf die abhängige Variable der Differenz des BSL-95-Wertes zwischen Zeitpunkt 2 und 3, $F(1,25)=8,998 ; \mathrm{p}=0,006 ; \mathrm{n}=27$. Bei einer Steigerung der Dysphorieverbesserung um 1 Einheit erhöht sich die anhaltende Wirkung um 4,9 Einheiten bezogen auf die Differenz der BSL-95-Werte zwischen Zeitpunkt 2 und 3. Dieses zweite Modell erklärt nur 24\% der Streuung bei einem starken Effekt nach Cohen von 1,03 (Tab. 6 und Tab. 7).

\section{Diskussion}

Unsere Daten zeigen als erstes keine signifikante Verschlechterung der BDI- und BSL-95-Werte zum Zeitpunkt 3 gegenüber dem Zeitpunkt 2, jedoch ist eine Verschlechterung zu vermuten, die al- 
lerdings aufgrund der begrenzten Patientenanzahl nicht mit einem Signifikanzniveau von $<0,05$ gezeigt werden konnte. Wir sehen zweitens jedoch eine fortbestehende Symptomverbesserung zu den späteren Messzeitpunkten bezogen auf den Therapiestart. Insgesamt sind die BSL-95-Werte von 157 zum letzten Zeitpunkt weiterhin hoch und mit einem Prozentrang von 30\% höher oder gleich der Normierungsstichprobe noch nicht zufriedenstellend. Damit dürften diese Ergebnisse auf eine anhaltende Symptomverbesserung nach Therapieende hinweisen.

Die Varianz des Katamnesezeitraums von 3 bis 51 Monaten ist problematisch zu sehen. Zum einen wären etwa ein Drittel der Patienten pro 17 Monate des Katamnesezeitraums statistisch zu erwarten gewesen, in die Auswertung flossen jedoch schon ein Drittel der Patienten im Katamnesezeitraum von 3-10 Monate direkt nach der Therapie ein. Dieser Effekt steht am ehesten im Zusammenhang mit einer über die Zeit abnehmenden ResponseRate. Die Prüfung, ob dieser Zeitfaktor die anhaltende Wirkung beeinflusst, war in einer linearen Regressionsanalyse nicht signifikant und ist somit für die vorliegenden Daten nicht als Erklärung zu sehen.

Unter der Annahme, dass Prädiktoren für Drop-out-Raten auch die Symptomverbesserung in der Studienpopulation beeinflussen könnten, haben wir ausgewählte Prädiktoren hierfür geprüft. So gilt die Anzahl früherer Suizidversuche in anderen Studien hinsichtlich der Drop-out-Rate als wichtiger Risikofaktor - entweder als Suizidalität [Rüsch et al., 2008] oder aber aufgrund sehr häufiger Suizidversuche (>9) in der Vergangenheit [Kröger et al., 2014]. Dieses konnten wir in unserer Vorstudie so nicht bestätigen bzw. in dieser Nachhaltigkeitsstudie ebenfalls nicht beschreiben.

Möglicherweise könnten ausgewählte Prädiktoren nur in kürzeren Katamnesezeiträumen, nicht aber in den längeren Katamnesezeiträumen relevant sein, und umgekehrt. Eine separate Auswertung wäre daher sinnvoll, aufgrund der dann jedoch geringen Fallgröße haben wir diese Untersuchung nicht durchgeführt, die in einer weiteren Studie sicherlich anzustreben wäre.

In der vorliegenden Studie gelang der Nachweis von Prädiktoren nur für 2 Modelle. Einzig die in der Subskala des BSL-95 genannte Verbesserung der «Dysphorie» kann etwa 25\% der anhaltenden Symptomverbesserung mit starkem Effekt bezogen auf die Skalen BDI und BSL erklären. In der BSL-95 wird «Dysphorie» durch schlechte Konzentration, jeweiligen Mangel an innerer Ruhe, Zuversichtlichkeit, Ausgeglichenheit und Unbeschwertheit, Tatkraft, Zuversicht, Zufriedenheit sowie Leitungsfähigkeit bzw. verminderte Ausgeruhtheit beschrieben.

Vorsichtigerweise könnte die Subskala «Dysphorie» gegebenenfalls noch als eine mögliche Autokorrelation zur BSL-95 diskutiert werden, in Bezug auf das BDI ist jedoch nicht davon auszugehen.

Insbesondere «Ärger und Zorn» als negative und spezifische Emotionen konnten in anderen Studien als Prädiktoren für höhere Drop-out-Raten der DBT herausgefiltert werden [Fassino et al., 2003; Rüsch et al., 2008]. Somit erscheint es diskutabel, dass eine Verbesserung dieser «Dysphorie», also eine erhöhte Ausgeglichenheit und Zufriedenheit während der Therapiephase, eine stärkere Nachhaltigkeit bewirkt, wohingegen Patienten mit negativen Emo- tionen im Extremfall auch schon höhere Drop-out-Raten haben. Verbesserte subjektive Zuversicht und Tatkraft und damit Leistungsfähigkeit sind entscheidende Prädiktoren für eine anhaltende Wirkung therapeutischer Interventionen, auch im Sinne des Wirkfaktormodells nach Grawe als Ressourcenaktivierung zu verstehen [Grawe, 2005]. Identitätsstörungen und Angst vor dem Verlassenwerden als Prädiktoren wie bei Yen et al. [2009] genannt, konnten wir in unserem Studiendesign nur mit den Messinstrumenten BSL95 und BDI allein nicht untersuchen.

In den letzten Jahren wurden Adhärenz und therapeutische Allianz als wichtig für einen guten Therapieerfolg beschrieben [Priebe et al., 2012; Tormoen et al., 2014]. Zu diskutieren ist, ob sich in unserer Risikogruppe, charakterisiert durch hohe «Dysphorie», die therapeutische Allianz gegebenenfalls vermindert hat und damit Therapieerfolge möglicherweise reduziert wurden. Diese Hypothese wäre aufgrund der selektierten dysfunktionalen Denk- und Verhaltensmuster und starken Emotionalität denkbar. Adhärenz bzw. therapeutische Allianz haben wir jedoch nicht gemessen, auch wenn aufgrund der Zertifizierung und des Bestehens eines tagesklinischen DBT-Angebotes über mehr als 5 Jahre eine hohe Identifizierung mit der DBT im Therapeutenteam zu erwarten ist [Swales et al., 2012] und damit dieses in guter Qualität und Stabilität vorliegen sollte. Adhärenz und therapeutische Allianz sind für weiterführende Studien jedoch zu fordern [Bedics et al., 2015].

Als ein anderer möglicher Einflussfaktor ist die eventuelle Medikation zwischen den Zeitpunkten 2 und 3 zu bedenken. Hierzu liegen keine Daten für unsere Studienpopulation nach Entlassung vor. Die uns bekannte Literatur bietet jedoch auch keine überzeugenden Hinweise, dass die Borderline-typische Symptomatik hierdurch gelindert werden könnte [Stoffers und Lieb, 2015].

Hinsichtlich erschwerender Komorbiditäten spielen in unserer Studienpopulation Suchterkrankungen, Essstörungen und PTBS als weitere in der Literatur bekannte Prädiktoren keine entscheidende Rolle, da therapiegefährdende Ausprägungen in der Diagnostikphase in hierfür spezialisierte Einrichtungen vermittelt wurden.

Auch die berufliche Eingliederung und Verbesserung sozialer Beziehungen erscheinen als Ziel und Therapieerfolg zur Stabilisation wichtig - unsere Daten bieten hierfür jedoch keine Auswertungsmöglichkeit. Wir können somit nicht darstellen, inwieweit tatsächlich das Gelingen einer Verbesserung in diesen wesentlichen Lebensbereichen die Verschlechterung oder Stabilität der Symptomatik vorhersagen könnte bzw. durch die DBT beeinflusst wurde. Auch die Berliner Borderline-Studie kann nach 1 Jahr ambulanter Therapie hierzu keine entsprechende Aussage treffen [Stiglmayr et al., 2014b].

\section{Limitationen}

Hinsichtlich unserer Studienergebnisse ist erstens festzuhalten, dass wir Daten einer unkontrollierten Studienpopulation vorfinden. Eine Kontrollgruppe ohne Therapieoption wäre über diesen langen Zeitraum bis zu 51 Monaten ethisch kaum umsetzbar, da die DBT mittlerweile ein überzeugendes Evidenzniveau erreicht hat, gegebenenfalls wäre ein Vergleich mit anderen spezifischen 
evidenzbasierten Psychotherapiemethoden denkbar [Stoffers et al., 2012]. Da keine Kontrollgruppe Verwendung fand, können die obigen Ergebnisse nicht die Wirkung der tagesklinischen DBT generell auf die BPS beschreiben, es könnte ja auch eine nicht untersuchte Gruppe mit einer BPS existieren, die im natürlichen Verlauf eine Symptombesserung erlangte.

Zweitens haben nur 50\% der Patienten zum Katamnesezeitpunkt geantwortet. Dies könnte einen Selektionseffekt darstellen und damit die dargestellten Ergebnisse verzerren. Vielleicht hat sich gerade die nicht antwortende Hälfte verschlechtert.

Drittens wären für eine mögliche Folgestudie Nachbeobachtungszeiträume in mehreren definierten Zeitabständen wünschenswert - die vorliegende Untersuchung erhebt jedoch Daten aus einem Nachbetrachtungszeitraum zwischen 3 und 51 Monaten, eine leider große zeitlichen Streuung.

Viertens würde eine höhere Rückantwortquote und damit größere Patientenanzahl auswertbare Ergebnisse besser untermauern.

Zusätzlich sollte der Katamneszeitaum besser beschreibbar sein, also welche therapeutischen Maßnahmen oder sozialen Veränderungen währenddessen erfolgten.

Ebenfalls lässt eine geringe Patientenzahl die differenzierte Subgruppenanalyse schnell an ihre Grenze geraten (z.B. Effekte psychotherapeutischer Anschlussbehandlung). Für robustere Ergebnisse wären deutlich größere Fallzahlen notwendig.

Schließlich handelt es sich hier um eine psychotherapeutische Anwendungsbeobachtung mit einem zusätzlichen Katamnesezeitpunkt. Einfache Urteile und Schlussfolgerungen sind nicht zulässig [Linden, 2017].

\section{Vorteile}

Aufgrund des naturalistischen Studiendesigns können wir Patientendaten jedoch direkt aus der täglichen Praxis auswerten. Hervorzuheben ist, dass wir im Vergleich zu anderen Studien über BPS Daten beider Geschlechter nutzen können. Eine mögliche Vorselektion ist sehr gering [Richter et al., 2014]. Wir können Verlaufsdaten präsentieren, die zu 3 wichtigen Zeitpunkten erhoben wurden (zu Beginn und zum Ende der Therapie sowie zu einem Katamnesezeitpunkt). Dieser Katamnesezeitraum erstreckt sich auf bis zu 51 Monate, im Mittel 22 Monate - ein langer Nachbetrach- tungszeitraum, den nur sehr wenige Studien so überhaupt untersuchten [Linehan et al., 2015].

\section{Zusammenfassung für die Praxis}

Die Ergebnisse dieser Anwendungsbeobachtung legen nahe, dass eine Symptombesserung durch eine 3-monatige tagesklinische DBT möglicherweise bis zu 51 Monate anhalten könnte - gemessen am BDI und mit der BSL-95. Weiter untersucht werden sollte, ob es Subgruppen der BPS gibt, deren anhaltende Therapiewirkung durch bestimmte psychologische Merkmale vielleicht schon während der Therapiephase verstärkt oder auch vermindert wird. Eine in dieser katamnestischen Nachuntersuchung beschriebene Subgruppe ist charakterisiert durch eine Verbesserung der «Dysphorie» während der Therapiephase mit einhergehender längerer Symptomverbesserung. Therapeuten sollten im Sinne eines Qualitätsmonitorings dem Merkmal «Dysphorie» aufmerksam begegnen. Möglicherweise ist die klassische DBT für diese Gruppe noch durch andere oder zusätzlich zu entwickelnde Interventionsstrategien zu erweitern.

\section{Dank}

Mein Dank gilt Sandra Schild und Genia Juschkewitsch von der Psychologischen Hochschule Berlin, die im Rahmen ihrer Masterarbeit die Datenerhebung übernahmen. Für die Beratung im Vorfeld der Studie und die Nutzung des NESTAP gilt mein ausdrücklicher Dank Prof. Eva-Lotta Brakemeier. Ohne die engagierte Arbeit des gesamten Therapeutenteams der Tagesklinik 1 im Vivantes Wenckebach-Krankenhaus wäre diese Arbeit nicht möglich geworden.

\section{Disclosure Statement}

Der Autor ist Facharzt für Psychiatrie und Psychotherapie mit Schwerpunkt Verhaltenstherapie und zertifizierter DBT-Therapeut nach den Richtlinien des Dachverbandes Dialektisch Behaviorale Therapie e.V. Er war als Vivantes-Mitarbeiter zum Zeitpunkt der Erhebung oberärztlich für die Etablierung des Konzepts der benannten Tagesklinik zuständig. Finanzielle Zuwendungen durch Pharmafirmen oder andere Personen/Institutionen, die in irgendeiner Weise diese Arbeit beeinflussen können, existieren nicht.

\section{Literatur}

Alvarez-Tomas I, Soler J, Bados A, Martin-Blanco A, Elices M, Carmona C, Bauza J, Pascual JC: Long-term course of borderline personality disorder: a prospective 10 year follow-up study. J Pers Disord 2017;31:590-605

Bedics JD, Atkins DC, Harned MS, Linehan MM: The therapeutic alliance as a predictor of outcome in dialectical behavior therapy versus nonbehavioral psychotherapy by experts for borderline personality disorder. Psychotherapy (Chic) 2015;52:67-77.

Bohus M, Kröger C: Psychopathologie und Psychotherapie der Borderline-Persönlichkeitsstörung: Zum gegenwärtigen Stand der Forschung. Nervenarzt 2011;82: $16-24$.

Brakemeier EL, Breger V, Spitzer C: Nebenwirkungen von ambulanter und stationärer Psychotherapie. VPP 2012; 44:489-510.
Coid J, Yang M, Tyrer P, Roberts A, Ullrich S: Prevalence and correlates of personality disorder in Great Britain. Br J Psychiatry 2006; 188:423-431.

Cristea IA, Gentili C, Cotet CD, Palomba D, Barbui C, Cuijpers P: Efficacy of psychotherapies for borderline personality disorder: a systematic review and metaanalysis. JAMA Psychiatry 2017;74:319-328.

Dams A, Schommer N, Ropke S, Heuser I, Lammers CH: Das Skilltraining und die poststationäre Effektivität der stationären dialektisch-behavioralen Therapie (DBT) nach sechs Monaten. Psychother Psychosom Med Psychol 2007;57:19-24.

Eikelmann B: Tagesklinische Behandlung in der Psychiatrie (Day hospital treatment in psychiatry). Nervenarzt 2010;81:355-363; quiz 364-355.
Fassino S, Abbate-Daga G, Piero A, Leombruni P, Rovera GG: Dropout from brief psychotherapy within a combination treatment in bulimia nervosa: role of personality and anger. Psychother Psychosom 2003;72:203210.

Grawe K: Empirisch validierte Wirkfaktoren statt Therapiemethoden. Report Psychologie 2005;311.

Gunderson JG, Stout RL, McGlashan TH, Shea MT, Morey LC, Grilo CM, Zanarini MC, Yen S, Markowitz JC, Sanislow C, Ansell E, Pinto A, Skodol AE: Ten-year course of borderline personality disorder: psychopathology and function from the Collaborative Longitudinal Personality Disorders study. Arch Gen Psychiatry 2011;68:827-837. 
Hautzinger M, Bailer M, Worall H: BDI Beck-DepressionsInventar Testhandbuch (German Version), ed 2. Bern, Verlag Hans Huber, 1995.

Kallert TW, Schutzwohl M, Matthes C: Aktuelle Strukturund Leistungsmerkmale allgemeinpsychiatrischer Tageskliniken in der Bunderepublik Deutschland (Current structural and procedural quality markers of psychiatric day hospitals in Germany). Psychiatr Prax 2003;30:72-82.

Kienast T, Stoffers J, Bermpohl F, Lieb K: Borderline personality disorder and comorbid addiction: epidemiology and treatment. Dtsch Arztebl Int 2014;111:280286.

Kleindienst N, Limberger MF, Schmahl C, Steil R, EbnerPriemer UW, Bohus M: Do improvements after inpatient dialectial behavioral therapy persist in the long term? A naturalistic follow-up in patients with borderline personality disorder. J Nerv Ment Dis 2008;196: 847-851.

Kleindienst N, Priebe K, Gorg N, Dyer A, Steil R, Lyssenko L, Winter D, Schmahl C, Bohus M: State dissociation moderates response to dialectical behavior therapy for posttraumatic stress disorder in women with and without borderline personality disorder. Eur J Psychotraumatol 2016;7:30375.

Kliem S, Kröger C, Kosfelder J: Dialectical behavior therapy for borderline personality disorder: a meta-analysis using mixed-effects modeling. J Consult Clin Psychol 2010;78:936-951.

Kröger C, Harbeck S, Rickert I, Wollburg W, Gersch K, Armbrust M, Kliem S: Remission, Response und deren Prädiktion nach einer dialektisch-behavioralen Therapie der Borderline-Persönlichkeitsstörung im stationären Setting. Z Klin Psychol Psychother 2013;42: 45-54.

Kröger C, Röpke S, Kliem S: Reasons for premature termination of dialectical behavior therapy for inpatients with borderline personality disorder. Behav Res Ther 2014;60:46-52.

Kröger C, Schweiger U, Sipos V, Arnold R, Kahl KG, Schunert T, Rudolf S, Reinecker H: Effectiveness of dialectical behaviour therapy for borderline personality disorder in an inpatient setting. Behav Res Ther 2006; 44:1211-1217.

- Kröger C, Schweiger U, Sipos V, Kliem S, Arnold R, Schunert T, Reinecker H: Dialectical behaviour therapy and an added cognitive behavioural treatment module for eating disorders in women with borderline personality disorder and anorexia nervosa or bulimia nervosa who failed to respond to previous treatments. An open trial with a 15-month follow-up. J Behav Ther Exp Psychiatry 2010;41:381-388.

Landes SJ, Chalker SA, Comtois KA: Predicting dropout in outpatient dialectical behavior therapy with patients with borderline personality disorder receiving psychiatric disability. Borderline Personal Disord Emot Dysregul 2016;3:9.

Lieb K, Völlm B, Rucker G, Timmer A, Stoffers JM: Pharmacotherapy for borderline personality disorder: Cochrane systematic review of randomised trials. $\mathrm{Br} J$ Psychiatry 2010;196:4-12.
Linden M: Psychosomatic inpatient rehabilitation: the German model. Psychother Psychosom 2014;83:205-212.

Linden M: Scientist-Practitioner-Gap und Anwendungsbeobachtungen. Verhaltenstherapie 2017;27:84-85.

Linehan MM, Korslund KE, Harned MS, Gallop RJ, Lungu A, Neacsiu AD, McDavid J, Comtois KA, MurrayGregory AM: Dialectical behavior therapy for high suicide risk in individuals with borderline personality disorder: a randomized clinical trial and component analysis. JAMA Psychiatry 2015;72:475-482.

Links PS, Kolla NJ, Guimond T, McMain S: Prospective risk factors for suicide attempts in a treated sample of patients with borderline personality disorder. Can J Psychiatry 2013;58:99-106.

Lynch TR, Trost WT, Salsman N, Linehan MM: Dialectical behavior therapy for borderline personality disorder. Annu Rev Clin Psychol 2007;3:181-205.

McMain SF, Guimond T, Streiner DL, Cardish RJ, Links PS: Dialectical behavior therapy compared with general psychiatric management for borderline personality disorder: clinical outcomes and functioning over a 2-year follow-up. Am J Psychiatry 2012;169:650-661.

Pasieczny N, Connor J: The effectiveness of dialectical behaviour therapy in routine public mental health settings: an Australian controlled trial. Behav Res Ther 2011;49:4-10.

Perroud N, Uher R, Dieben K, Nicastro R, Huguelet P: Predictors of response and drop-out during intensive dialectical behavior therapy. J Pers Disord 2010;24:634650.

Priebe S, Bhatti N, Barnicot K, Bremner S, Gaglia A, Katsakou C, Molosankwe I, McCrone P, Zinkler M: Effectiveness and cost-effectiveness of dialectical behaviour therapy for self-harming patients with personality disorder: a pragmatic randomised controlled trial. Psychother Psychosom 2012;81:356-365.

Richter C, Heinemann B, Kehn M, Steinacher B: Effektivität der dialektisch-behavioralen Therapie (DBT) in der tagesklinischen Behandlung der Borderline-Persönlichkeitsstörung: Bedeutung von Medikation und Behandlungskosten (Effectiveness of dialectical behavior therapy (DBT) in an outpatient clinic for borderline personality disorders - impact of medication use and treatment costs). Psychiatr Prax 2014;41:148-152.

Rüsch N, Schiel S, Corrigan PW, Leihener F, Jacob GA, Olschewski M, Lieb K, Bohus M: Predictors of dropout from inpatient dialectical behavior therapy among women with borderline personality disorder. J Behav Ther Exp Psychiatry 2008;39:497-503.

Sansone RA, Wiederman MW: The abuse of prescription medications: borderline personality patients in psychiatric versus non-psychiatric settings. Int J Psychiatry Med 2009;39:147-154.

Steuwe C, Berg M, Driessen M, Beblo T: Impact of therapist change after initial contact and traumatic burden on dropout in a naturalistic sample of inpatients with borderline pathology receiving dialectical behavior therapy. Borderline Personal Disord Emot Dysregul 2017;4:14.
Stiglmayr C: Spannung und Dissoziation bei der Borderline-Persönlichkeitsstörung. Frankfurt/M., Verlag Peter Lang, 2003.

Stiglmayr C, Stecher-Mohr J, Wagner T, Meibetaner J, Spretz D, Steffens C, Roepke S, Fydrich T, SalbachAndrae H, Schulze J, Renneberg B: Effectiveness of dialectic behavioral therapy in routine outpatient care: the Berlin Borderline Study. Borderline Personal Disord Emot Dysregul 2014a;1:20.

Stiglmayr C, Stecher-Mohr J, Wagner T, Meißner J, Spretz D, Steffens C, Röpke S, Fydrich T, Salbach-Andrae H, Schulze J, Renneberg B: Effectiveness of dialectic behavioral therapy in routine outpatient care: the Berlin Borderline Study. Borderline Personal Disord Emot Dysregul 2014b;1:1-11.

Stoffers JM, Lieb K: Pharmacotherapy for borderline personality disorder - current evidence and recent trends. Curr Psychiatry Rep 2015;17:534.

Stoffers JM, Völlm BA, Rücker G, Timmer A, Huband N, Lieb K: Psychological therapies for people with borderline personality disorder. Cochrane Database Syst Rev 2012;(8):CD005652.

Stone MH: Long-term course of borderline personality disorder. Psychodyn Psychiatry 2016;44:449-474.

Stone MH: Borderline patients: 25 to 50 years later: with commentary on outcome factors. Psychodyn Psychiatry 2017;45:259-296.

Swales MA, Taylor B, Hibbs RA: Implementing dialectical behaviour therapy: programme survival in routine healthcare settings. J Ment Health 2012;21:548-555.

Torgersen S, Lygren S, Oien PA, Skre I, Onstad S, Edvardsen J, Tambs K, Kringlen E: A twin study of personality disorders. Compr Psychiatry 2000;41:416-425.

Tormoen AJ, Groholt B, Haga E, Brager-Larsen A, Miller A, Walby F, Stanley B, Mehlum L: Feasibility of dialectical behavior therapy with suicidal and self-harming adolescents with multi-problems: training, adherence, and retention. Arch Suicide Res 2014;18:432-444.

Trull TJ, Jahng S, Tomko RL, Wood PK, Sher KJ: Revised NESARC personality disorder diagnoses: gender, prevalence, and comorbidity with substance dependence disorders. J Pers Disord 2010;24:412-426.

van den Bosch LM, Sinnaeve R, Hakkaart-van Roijen L, van Furth EF: Efficacy and cost-effectiveness of an experimental short-term inpatient dialectical behavior therapy (DBT) program: study protocol for a randomized controlled trial. Trials 2014;15:152.

Yen S, Johnson J, Costello E, Simpson EB: A 5-day dialectical behavior therapy partial hospital program for women with borderline personality disorder: predictors of outcome from a 3-month follow-up study. J Psychiatr Pract 2009;15:173-182.

Zanarini MC, Frankenburg FR, Dubo ED, Sickel AE, Trikha A, Levin A, Reynolds V: Axis I comorbidity of borderline personality disorder. Am J Psychiatry 1998; 155:1733-1739.

Zentralinstitut für Seelische Gesundheit (ZfS): Borderline Symptom-Liste 95 (BSL-95). Mannheim, Zfs, 2014. 